\title{
Author Index Volume 32 (2012)
}

The issue number is given in front of the pagination

Abdel-All, Z., see Mukaetova-Ladinska, E.B. (2) 431436

Abe, K., see Wu, J. (2) 317-328

Ahn, H.J., see Cortes-Canteli, M. (3) 599-608

Altamura, C., see Silvestrini, M. (3) 689-698

Ambrose, C.T., Neuroangiogenesis: A Vascular Basis for Alzheimer's Disease and Cognitive Decline During Aging (3) 773-788

Anand, K.S., see Dhikav, V. (3) 711-718

Andrade, J., see Mukaetova-Ladinska, E.B. (2) 431436

Andreasen, N., see Höglund, K. (4) 939-947

Andreasen, N., see Olsson, B. (2) 273-276

Angeli, A., see Kervern, M. (1) 183-196

Annas, P., see Höglund, K. (4) 939-947

Annas, P., see Olsson, B. (2) 273-276

Arai, H., see Waragai, M. (1) 33-41

Arancio, O., see Hashimoto, G. (2) 437-445

Arendash, G.W., Transcranial Electromagnetic Treatment Against Alzheimer's Disease: Why it has the Potential to Trump Alzheimer's Disease Drug Development (2) 243-266

Arnold, S.E., see Zhang, Q. (3) 677-687

Asada, T., see Yasuno, F. (4) 895-903

Asthana, S., see Wharton, W. (1) 147-156

Aubert, I., see Girão da Cruz, M.T. (2) 267-272

Auriacombe, S., see Wallon, D. (1) 19-22

Ayala-Grosso, C.A., see Girão da Cruz, M.T. (2) 267272

Aziz, F., see Hashimoto, G. (2) 437-445

Aziz, A., see Höglund, K. (4) 939-947

Baciu, M., see Klein-Koerkamp, Y. (1) 109-125

Bagnoli, S., see Tedde, A. (1) 13-17

Bai, F., Y. Shi, Y. Yuan, C. Yue, L. Zhuang, X. Xu, $X$. Liu and Z. Zhang, Association of a GSK-3 $\beta$ Polymorphism with Brain Resting-State Function in Amnestic-Type Mild Cognitive Impairment (2) 387-396

Baig, I., see Beckett, T.L. (1) 127-138

Balucani, C., see Silvestrini, M. (3) 689-698

Banks, W.A., see Farr, S.A. (2) 447-455
Baranowska, B., see Gaj, P. (1) 157-168

Baranowska-Bik, A., see Gaj, P. (1) 157-168

Barcikowska, M., see Gaj, P. (1) 157-168

Barone, E., see Domenico, F.D. (2) 277-289

Barr, R.K., see Bharadwaj, P.R. (4) 949-967

Barua, N.U., J.S. Miners, A.S. Bienemann, M.J. Wyatt, K. Welser, A.B. Tabor, H.C. Hailes, S. Love and S.S. Gill, Convection-Enhanced Delivery of Neprilysin: A Novel Amyloid- $\beta$-Degrading Therapeutic Strategy (1) 43-56

Basun, H., see Höglund, K. (4) 939-947

Basun, H., see Olsson, B. (2) 273-276

Bayer, A., see Tales, A. (2) 457-466

Bayod, S., see del Valle, J. (1) 233-240

Beas-Zárate, C., see del Valle, J. (1) 233-240

Beaudoin, M., see Klein-Koerkamp, Y. (1) 109-125

Becker, J.T., see Choi, S.H. (4) 1043-1049

Beckett, T.L., R.L. Webb, D.M. Niedowicz, C.J. Holler, S. Matveev, I. Baig, H. LeVine III, J.N. Keller and M.P. Murphy, Postmortem Pittsburgh Compound B (PiB) Binding Increases with Alzheimer's Disease Progression (1) 127-138

Beckett, T.L., see Head, E. (4) 1029-1042

Bell, R.D., The Imbalance of Vascular Molecules in Alzheimer's Disease (3) 699-709

Bennett, D.A., see Shah, R.C. (3) 579-586

Berg, D., see Maetzler, W. (1) 95-100

Bethel, S.R., see Head, E. (4) 1029-1042

Bharadwaj, P.R., G. Verdile, R.K. Barr, V. Gupta, J.W. Steele, M.L. Lachenmayer, Z. Yue, M.E. Ehrlich, G. Petsko, S. Ju, D. Ringe, S.E. Sankovich, J.M. Caine, I.G. Macreadie, S. Gandy and R.N. Martins, Latrepirdine (Dimebon ${ }^{\mathrm{TM}}$ ) Enhances Autophagy and Reduces Intracellular GFP-A $\beta_{42}$ Levels in Yeast (4) 949-967

Bialopiotrowicz, E., A. Szybinska, B. Kuzniewska, L. Buizza, D. Uberti, J. Kuznicki and U. Wojda, Highly Pathogenic Alzheimer's Disease Presenilin 1 P117R Mutation Causes a Specific Increase in p53 and p21 Protein Levels and Cell Cycle Dysregulation in Human Lymphocytes (2) 397-415 
Bienemann, A.S., see Barua, N.U. (1) 43-56

Bik, W., see Gaj, P. (1) 157-168

Bimonte-Nelson, H., see Wolf, A.B. (1) 217-232

Bitel, C.L., C. Kasinathan, R.H. Kaswala, W.L. Klein and P.H. Frederikse, Amyloid- $\beta$ and Tau Pathology of Alzheimer's Disease Induced by Diabetes in a Rabbit Animal Model (2) 291-305

Biundo, F., see Lanni, C. (4) 919-926

Blennow, K., see Höglund, K. (4) 939-947

Blennow, K., see Olsson, B. (2) 273-276

Bogstedt, A., see Höglund, K. (4) 939-947

Bompas, A., see Tales, A. (2) 457-466

Bottino, C.M.C., see Lopes, M.A. (2) 307-316

Bouras, C., see Costanza, A. (3) 643-652

Braden, B.B., see Wolf, A.B. (1) 217-232

Bradley, M.A., see Lovell, M.A. (1) 139-146

Bresch, F., see Head, E. (4) 1029-1042

Brockmann, K., see Maetzler, W. (1) 95-100

Bruchey, A.K., see Rojas, J.C. (3) 741-752

Buisson, A., see Kervern, M. (1) 183-196

Buizza, L., see Bialopiotrowicz, E. (2) 397-415

Burns, J.M., see Royall, D.R. (2) 467-478

Burrell, J., see Leyton, C.E. (4) 1051-1059

Butterfield, D.A., see Domenico, F.D. (2) 277-289

Cahill, L.F., see Segal, S.K. (4) 1011-1018

Caine, J.M., see Bharadwaj, P.R. (4) 949-967

Camins, A., see del Valle, J. (1) 233-240

Campion, D., see Wallon, D. (1) 19-22

Carlsson, C.M., see Wharton, W. (1) 147-156

Casarejos, M.J., see Solano, R.M. (1) 57-76

Cedazo-Minguez, A., see Maioli, S. (2) 341-355

Cha, R.H., see Roberts, R.O. (2) 329-339

Chapa, R.P., see Fawver, J.N. (1) 197-215

Chen, Q., see Wang, X. (1) 77-94

Cho, Z.-H., see Kim, M.-J. (4) 927-938

Chodakowska-Żebrowska, M., see Gaj, P. (1) 157-168

Choi, S.H., M. Olabarrieta, O.L. Lopez, V. Maruca, S.T. DeKosky, R.L. Hamilton, J.T. Becker for the Alzheimer's Disease Research Center, Gray Matter Atrophy Associated with Extrapyramidal Signs in the Lewy Body Variant of Alzheimer's Disease (4) 1043-1049

Coccia, R., see Domenico, F.D. (2) 277-289

Cocciolo, A., see Domenico, F.D. (2) 277-289

Collins, O., see Kennelly, S. (3) 609-621

Colom, L.V., see Fawver, J.N. (1) 197-215

Cortes-Canteli, M., D. Zamolodchikov, H.J. Ahn, S. Strickland and E.H. Norris, Fibrinogen and Altered Hemostasis in Alzheimer's Disease (3) 599-608

Coskuner, O., see Fawver, J.N. (1) 197-215
Costanza, A., A. Xekardaki, E. Kövari, G. Gold, C. Bouras and P. Giannakopoulos, Microvascular Burden and Alzheimer-Type Lesions Across the Age Spectrum (3) 643-652

Cotman, C.W., see Segal, S.K. (4) 1011-1018

Cummings, S.R., see Tranah, G.J. (2) 357-372

Cuzzoni, G., see Lanni, C. (4) 919-926

Dąbrowska, M., see Gaj, P. (1) 157-168

Dantoine, T., see Fehlbaum-Beurdeley, P. (1) 169-181

Das, S., see Raychaudhuri, M. (2) 479-493

DaSilva, K.A., see Girão da Cruz, M.T. (2) 267-272

de Bem, A.F., see Moreira, E.L.G. (2) 495-511

de la Torre, J.C., Cerebral Hemodynamics and Vascular

Risk Factors: Setting the Stage for Alzheimer's Disease (3) 553-567

de la Torre, J.C., Physiopathology of Vascular Risk Factors in Alzheimer's Disease (3) 517-518

de Laat, K.F., see Gons, R.A.R. (3) 623-631

de Laat, K.F., see van Norden, A.G.W. (3) 667-676

de Leeuw, F.-E., see Gons, R.A.R. (3) 623-631

de Leeuw, F.-E., see van Norden, A.G.W. (3) 667-676

de Oliveira, J., see Moreira, E.L.G. (2) 495-511

de Yébenes, J.G., see Solano, R.M. (1) 57-76

Dean, R.A., see Lachno, D.R. (4) 905-918

Dean, R.A., see Yu, P. (2) 373-385

DeKosky, S.T., see Choi, S.H. (4) 1043-1049

del Valle, J., S. Bayod, A. Camins, C. Beas-Zárate, D.A. Velázquez-Zamora, I. González-Burgos and M. Pallás, Dendritic Spine Abnormalities in Hippocampal CA1 Pyramidal Neurons Underlying Memory Deficits in the SAMP8 Mouse Model of Alzheimer's Disease (1) 233-240

Deramecourt, V., see Wallon, D. (1) 19-22

Désiré, L., see Fehlbaum-Beurdeley, P. (1) 169-181

Desobry, K., see Sanchez, A. (3) 587-597

Detre, J.A., see Zhang, Q. (3) 677-687

Dhikav, V., and K.S. Anand, Are Vascular Factors Linked to the Development of Hippocampal Atrophy in Alzheimer's Disease? (3) 711-718

Ding, D., see Zhao, Q. (4) 1019-1027

Domenico, F.D., E. Barone, C. Mancuso, M. Perluigi, A. Cocciolo, P. Mecocci, D.A. Butterfield and R. Coccia, HO-1/BVR-A System Analysis in Plasma from Probable Alzheimer's Disease and Mild Cognitive Impairment Subjects: A Potential Biochemical Marker for the Prediction of the Disease (2) 277-289

Dowling, A.L.S., see Head, E. (4) 1029-1042

Dowling, M., see Wharton, W. (1) 147-156 
Duong, K.T., see Fawver, J.N. (1) 197-215

Dutar, P., see Kervern, M. (1) 183-196

Ehrlich, M.E., see Bharadwaj, P.R. (4) 949-967

Einstein, R., see Fehlbaum-Beurdeley, P. (1) 169-181

Emerson, J.K., see Lachno, D.R. (4) 905-918

Engler-Chiurazzi, E., see Wolf, A.B. (1) 217-232

Ercal, N., see Farr, S.A. (2) 447-455

Eschweiler, G.W., see Maetzler, W. (1) 95-100

Escudero, J., E. Ifeachor, J.P. Zajicek and for the Alzheimer's Disease Neuroimaging Initiative, Bioprofile Analysis: A New Approach for the Analysis of Biomedical Data in Alzheimer's Disease (4) 997-1010

Fabre, S., see Höglund, K. (4) 939-947

Farina, M., see Moreira, E.L.G. (2) 495-511

Farr, S.A., T.O. Price, W.A. Banks, N. Ercal and J.E. Morley, Effect of Alpha-Lipoic Acid on Memory, Oxidation, and Lifespan in SAMP8 Mice (2) 447 455

Fawver, J.N., K.T. Duong, O. Wise-Scira, R.P. Chapa, H.E. Schall, O. Coskuner, X. Zhu, L.V. Colom and Ian V.J. Murray, Probing and Trapping a Sensitive Conformation: Amyloid- $\beta$ Fibrils, Oligomers, and Dimers (1) 197-215

Fehlbaum-Beurdeley, P., O. Sol, L. Désiré, J. Touchon, T. Dantoine, M. Vercelletto, A. Gabelle, A.C. Jarrige, R. Haddad, J.C. Lemarié, W. Zhou, H. Hampel, R. Einstein, B. Vellas and on behalf of the EHTAD/002 study group, Validation of AclarusDx ${ }^{\mathrm{TM}}$, a BloodBased Transcriptomic Signature for the Diagnosis of Alzheimer's Disease (1) 169-181

Feldstein, C.A., Association Between Chronic Blood Pressure Changes and Development of Alzheimer's Disease (3) 753-763

Ferrioli, E., see Lopes, M.A. (2) 307-316

Fister, S.X., see Lovell, M.A. (1) 139-146

Flores, B. and R. von Bernhardi, Transforming Growth Factor $\beta 1$ Modulates Amyloid $\beta$-Induced Glial Activation Through the Smad3-Dependent Induction of MAPK Phosphatase-1 (2) 417-429

Foxely, S., see Little, D.M. (1) 101-107

Frederikse, P.H., see Bitel, C.L. (2) 291-305

Froestl, W., A. Muhs and A. Pfeifer, Cognitive Enhancers (Nootropics). Part 1: Drugs Interacting with Receptors (4) 793-887

Fusari, L., see Maioli, S. (2) 341-355

Gabelle, A., see Fehlbaum-Beurdeley, P. (1) 169-181 Gaenslen, A., see Maetzler, W. (1) 95-100
Gaj, P., A. Paziewska, W. Bik, M. Dąbrowska, A. Baranowska-Bik,M.Styczynska,M.ChodakowskaŻebrowska, A. Pfeffer-Baczuk, M. Barcikowska, B. Baranowska and J. Ostrowski, Identification of a Late Onset Alzheimer's Disease Candidate Risk Variant at 9q21.33 in Polish Patients (1) 157-168

Gandy, S., see Bharadwaj, P.R. (4) 949-967

Garbin, G., see Lanni, C. (4) 919-926

Garcia, A.N., see Wolf, A.B. (1) 217-232

Gay, D., see Sanchez, A. (3) 587-597

Geda, Y.E., see Roberts, R.O. (2) 329-339

Giannakopoulos, P., see Costanza, A. (3) 643-652

Gil-Bea, F., see Maioli, S. (2) 341-355

Gill, S.S., see Barua, N.U. (1) 43-56

Girão da Cruz, M.T., J. Jordão, K.A. DaSilva, C.A. Ayala-Grosso, A. Ypsilanti, Y.-Q. Weng, F.M. LaFerla, J. McLaurin and I. Aubert, Early Increases in Soluble Amyloid- $\beta$ Levels Coincide with Cholinergic Degeneration in 3xTg-AD Mice (2) 267-272

Glatzel, M., see Sepulveda-Falla, D. (1) 1-12

Gleason, C.E., see Wharton, W. (1) 147-156

Godau, J., see Maetzler, W. (1) 95-100

Gold, G., see Costanza, A. (3) 643-652

Gómez, A., see Solano, R.M. (1) 57-76

Gons, R.A.R., L.J.B. van Oudheusden, K.F. de Laat, A.G.W. van Norden, I.W.M. van Uden, D.G. Norris, M.P. Zwiers, E. van Dijk and F.-E. de Leeuw, Hypertension is Related to the Microstructure of the Corpus Callosum: The RUN DMC Study (3) 623-631

Gonzales, C., see Lachno, D.R. (4) 905-918

González-Burgos, I., see del Valle, J. (1) 233-240

Gonzalez-Lima, F., see Rojas, J.C. (3) 741-752

Govoni, S., see Lanni, C. (4) 919-926

Grammas, P., see Sanchez, A. (3) 587-597

Grossheim, M., see Head, E. (4) 1029-1042

Guo, Q., see Zhao, Q. (4) 1019-1027

Gupta, V., see Bharadwaj, P.R. (4) 949-967

Haddad, R., see Fehlbaum-Beurdeley, P. (1) 169-181

Hailes, H.C., see Barua, N.U. (1) 43-56

Hall, S.D., see Yu, P. (2) 373-385

Hamilton, R.L., see Choi, S.H. (4) 1043-1049

Hampel, H., see Fehlbaum-Beurdeley, P. (1) 169-181

Hannequin, D., see Wallon, D. (1) 19-22

Harris, T.B., see Tranah, G.J. (2) 357-372

Hashimoto, G., M. Sakurai, A.F. Teich, F. Saeed, F. Aziz and O. Arancio, 5- $\mathrm{HT}_{4}$ Receptor Stimulation Leads to Soluble A $\beta P P \alpha$ Production Through MMP-9 Upregulation (2) 437-445 
Hashimoto, M., see Waragai, M. (1) 33-41

Haworth, J., see Tales, A. (2) 457-466

Head, E., H.L. Murphey, A.L.S. Dowling, K.L. McCarty, S.R. Bethel, J.A. Nitz, M. Pleiss, J. Vanrooyen, M. Grossheim, J.R. Smiley, M.P. Murphy, T.L. Beckett, D. Pagani, F. Bresch and C. Hendrix, A Combination Cocktail Improves Spatial Attention in a Canine Model of Human Aging and Alzheimer's Disease (4) 1029-1042

Hendrix, C., see Head, E. (4) 1029-1042

Hodges, J.R., see Leyton, C.E. (4) 1051-1059

Höglund, K., A. Bogstedt, S. Fabre, A. Aziz, P. Annas, H. Basun, L. Minthon, L. Lannfelt, K. Blennow and N. Andreasen, Longitudinal Stability Evaluation of Biomarkers and Their Correlation in Cerebrospinal Fluid and Plasma from Patients with Alzheimer's Disease (4) 939-947

Höglund, K., see Olsson, B. (2) 273-276

Holler, C.J., see Beckett, T.L. (1) 127-138

Honea, R.A., see Royall, D.R. (2) 467-478

Hong, Z., see Zhao, Q. (4) 1019-1027

Hot, P., see Klein-Koerkamp, Y. (1) 109-125

Huang, H.-C., K. XuandZ.-F. Jiang, Curcumin-Mediated Neuroprotection Against Amyloid- $\beta$-Induced Mitochondrial Dysfunction Involves the Inhibition of GSK-3ß (4) 981-996

Huber, H., see Maetzler, W. (1) 95-100

Ifeachor, E., see Escudero, J. (4) 997-1010

Igarashi, K., see Waragai, M. (1) 33-41

Ikejima, C., see Yasuno, F. (4) 895-903

Iwai, N., see Waragai, M. (1) 33-41

Jacobson, L.E., see Wharton, W. (1) 147-156

James, P.W., see Mukaetova-Ladinska, E.B. (2) 431436

Jarrige, A.C., see Fehlbaum-Beurdeley, P. (1) 169-181

Jeon, H.-A., see Kim, M.-J. (4) 927-938

Jiang, Z.-F., see Huang, H.-C. (4) 981-996

Johnson, S.C., see Wharton, W. (1) 147-156

Jordão, J., see Girão da Cruz, M.T. (2) 267-272

Ju, S., see Bharadwaj, P.R. (4) 949-967

Kalaria, R.N., see Mukaetova-Ladinska, E.B. (2) 431436

Kasahata, N., T. Uchihara, S. Orimo, A. Nakamura and Y. Makita, Limbic and Nigral Lewy Bodies and Alzheimer's Disease Pathology Mimicking Progressive Supranuclear Palsy in a 75-Year-Old Man with Preserved Cardiac Uptake of MIBG (4) 889-894
Kashiwagi, K., see Waragai, M. (1) 33-41

Kasinathan, C., see Bitel, C.L. (2) 291-305

Kaswala, R.H., see Bitel, C.L. (2) 291-305

Katzman, S.M., see Tranah, G.J. (2) 357-372

Keller, J.N., see Beckett, T.L. (1) 127-138

Kennelly, S. and O. Collins, Walking the Cognitive "Minefield" Between High and Low Blood Pressure (3) 609-621

Kervern, M., A. Angeli, O. Nicole, F. Léveillé, B. Parent, V. Villette, A. Buisson and P. Dutar, Selective Impairment of Some Forms of Synaptic Plasticity by Oligomeric Amyloid- $\beta$ Peptide in the Mouse Hippocampus: Implication of Extrasynaptic NMDA Receptors (1) 183-196

Kim, M.-J., K.-M. Lee, Y.-D. Son, H.-A. Jeon, Y.-B. Kim and Z.-H. Cho, Increased Basal Forebrain Metabolism in Mild Cognitive Impairment: An Evidence for Brain Reserve in Incipient Dementia (4) $927-938$

Kim, Y.-B., see Kim, M.-J. (4) 927-938

Kivipelto, M., see Tolppanen, A.-M. (3) 531-540

Klein, W.L., see Bitel, C.L. (2) 291-305

Klein-Koerkamp, Y., M. Beaudoin, M. Baciu and P. Hot, Emotional Decoding Abilities in Alzheimer's Disease: A Meta-Analysis (1) 109-125

Knopman, D.S., see Roberts, R.O. (2) 329-339

Kodama, C., see Yasuno, F. (4) 895-903

Koikkalainen, J., see Mattila, J. (4) 969-979

Konrad, R.J., see Lachno, D.R. (4) 905-918

Korcarz, C., see Wharton, W. (1) 147-156

Kövari, E., see Costanza, A. (3) 643-652

Kusne, Y., see Wolf, A.B. (1) 217-232

Kuznicki, J., see Bialopiotrowicz, E. (2) 397-415

Kuzniewska, B., see Bialopiotrowicz, E. (2) 397-415

Lachenmayer, M.L., see Bharadwaj, P.R. (4) 949-967

Lachno, D.R., J.K. Emerson, H. Vanderstichele, C. Gonzales, F. Martényi, R.J. Konrad, J.A. Talbot, S.L. Lowe, P.E. Oefinger and R.A. Dean, Validation of a Multiplex Assay for Simultaneous Quantification of Amyloid- $\beta$ Peptide Species in Human Plasma with Utility for Measurements in Studies of Alzheimer's Disease Therapeutics (4) 905-918

LaFerla, F., see Wolf, A.B. (1) 217-232

LaFerla, F.M., see Girão da Cruz, M.T. (2) 267-272

Lam, E.T., see Tranah, G.J. (2) 357-372

Langkamp, M., see Maetzler, W. (1) 95-100

Lannfelt, L., see Höglund, K. (4) 939-947

Lannfelt, L., see Olsson, B. (2) 273-276 
Lanni, C., G. Garbin, A. Lisa, F. Biundo, A. Ranzenigo, E. Sinforiani, G. Cuzzoni, S. Govoni, G.N. Ranzani and M. Racchi, Influence of COMT Val158Met Polymorphism on Alzheimer's Disease and Mild Cognitive Impairment in Italian Patients (4) 919926

Lazarov, O., see Little, D.M. (1) 101-107

Le Ber, I., see Wallon, D. (1) 19-22

Lee, K.-M., see Kim, M.-J. (4) 927-938

Lemarié, J.C., see Fehlbaum-Beurdeley, P. (1) 169-181

Leonards, U., see Tales, A. (2) 457-466

Lerche, S., see Maetzler, W. (1) 95-100

Leurgans, S., see Shah, R.C. (3) 579-586

Léveillé, F., see Kervern, M. (1) 183-196

LeVine III, H., see Beckett, T.L. (1) 127-138

Leyton, C.E., O. Piguet, S. Savage, J. Burrell and J.R. Hodges, The Neural Basis of Logopenic Progressive Aphasia (4) 1051-1059

Li, Q., see Wu, J. (2) 317-328

Lisa, A., see Lanni, C. (4) 919-926

Little, D.M., S. Foxely and O. Lazarov, A Preliminary Study Targeting Neuronal Pathways Activated Following Environmental Enrichment by Resting State Functional Magnetic Resonance Imaging (1) 101-107

Litvoc, J., see Lopes, M.A. (2) 307-316

Liu, X., see Bai, F. (2) 387-396

Liu, Y., see Tranah, G.J. (2) 357-372

Lombardo, N.E., see Wolf, A.B. (1) 217-232

Lopera, F., see Sepulveda-Falla, D. (1) 1-12

Lopes, M.A., E. Ferrioli, E.Y. Nakano, J. Litvoc and C.M.C. Bottino, High Prevalence of Dementia in a Community-Based Survey of Older People from Brazil: Association with Intellectual Activity Rather than Education (2) 307-316

Lopez, O.L., see Choi, S.H. (4) 1043-1049

Lötjönen, J., see Mattila, J. (4) 969-979

Love, S., see Barua, N.U. (1) 43-56

Lovell, M.A., M.A. Bradley and S.X. Fister, 4-Hydroxyhexenal (HHE) Impairs Glutamate Transport in Astrocyte Cultures (1) 139-146

Lowe, S.L., see Lachno, D.R. (4) 905-918

Lucenteforte, E., see Tedde, A. (1) 13-17

Lue, L., see Wolf, A.B. (1) 217-232

Luo, J., see Sanchez, A. (3) 587-597

Luzzi, S., see Silvestrini, M. (3) 689-698

Macreadie, I.G., see Bharadwaj, P.R. (4) 949-967

Maetzler, W., M. Langkamp, S. Lerche, J. Godau, K. Brockmann, A. Gaenslen, H. Huber, I. Wurster, R. Niebler, G.W. Eschweiler and D. Berg, Lowered
Serum Amyloid- $\beta_{1-42}$ Autoantibodies in Individuals with Lifetime Depression (1) 95-100

Maioli, S., E. Puerta, P. Merino-Serrais, L. Fusari, F. Gil-Bea, R. Rimondini and A. Cedazo-Minguez, Combination of Apolipoprotein E4 and High Carbohydrate Diet Reduces Hippocampal BDNF and Arc Levels and Impairs Memory in Young Mice (2) 341-355

Makita, Y., see Kasahata, N. (4) 889-894

Malmeström, C., see Olsson, B. (2) 273-276

Mancuso, C., see Domenico, F.D. (2) 277-289

Marshall, R.S., Effects of Altered Cerebral Hemodynamics on Cognitive Function (3) 633-642

Martényi, F., see Lachno, D.R. (4) 905-918

Martenyi, F., see Yu, P. (2) 373-385

Martinez, J., see Sanchez, A. (3) 587-597

Martins, R.N., see Bharadwaj, P.R. (4) 949-967

Maruca, V., see Choi, S.H. (4) 1043-1049

Mattila, J., H. Soininen, J. Koikkalainen, D. Rueckert, R. Wolz, G. Waldemar, J. Lötjönen and for the Alzheimer's Disease Neuroimaging Initiative, Optimizing the Diagnosis of Early Alzheimer's Disease in Mild Cognitive Impairment Subjects (4) 969-979

Matveev, S., see Beckett, T.L. (1) 127-138

McCarty, K.L., see Head, E. (4) 1029-1042

McLaurin, J., see Girão da Cruz, M.T. (2) 267-272

McNally, R.J.Q., see Mukaetova-Ladinska, E.B. (2) 431-436

Mecocci, P., see Domenico, F.D. (2) 277-289

Mecocci, P., see Polidori, M.C. (3) 521-530

Mena, M.A., see Solano, R.M. (1) 57-76

Merino-Serrais, P., see Maioli, S. (2) 341-355

Miners, J.S., see Barua, N.U. (1) 43-56

Minthon, L., see Höglund, K. (4) 939-947

Mizoi, M., see Waragai, M. (1) 33-41

Mizukami, K., see Yasuno, F. (4) 895-903

Moll van Charante, E.P., see Richard, E. (3) 733-740

Mooney, S., see Tranah, G.J. (2) 357-372

Moreira, E.L.G., J. de Oliveira, J.C. Nunes, D.B. Santos, F.C. Nunes, D.S.C. Vieira, R.M. Ribeirodo-Valle, F.A. Pamplona, A.F. de Bem, M. Farina, R. Walz and R.D. Prediger, Age-Related Cognitive Decline in Hypercholesterolemic LDL Receptor Knockout Mice $\left(\mathrm{LDLr}^{--}\right)$: Evidence of Antioxidant Imbalance and Increased Acetylcholinesterase Activity in the Prefrontal Cortex (2) 495-511

Morley, J.E., see Farr, S.A. (2) 447-455

Moses, G.S.D., see Wolf, A.B. (1) 217-232

Muhs, A., see Froestl, W. (4) 793-887 
Mukaetova-Ladinska, E.B., Z. Abdel-All, J. Andrade, R.J.Q. McNally, P.W. James, R.N. Kalaria and J.T. O'Brien, Increase in Platelet Immunoglobulin in Alzheimer's Disease is Normalised Following Cholinesterase Inhibitor Treatment: Preliminary Results (2) 431-436

Mukhopadhyay, D., see Raychaudhuri, M. (2) 479-493

Murphey, H.L., see Head, E. (4) 1029-1042

Murphy, M.P., see Beckett, T.L. (1) 127-138

Murphy, M.P., see Head, E. (4) 1029-1042

Murray, Ian V.J., see Fawver, J.N. (1) 197-215

Nacmias, B., see Tedde, A. (1) 13-17

Nakamura, A., see Kasahata, N. (4) 889-894

Nakamura, N., see Wu, J. (2) 317-328

Nakano, E.Y., see Lopes, M.A. (2) 307-316

Nalls, M.A., see Tranah, G.J. (2) 357-372

Newman, A.B., see Tranah, G.J. (2) 357-372

Nicole, O., see Kervern, M. (1) 183-196

Niebler, R., see Maetzler, W. (1) 95-100

Niedowicz, D.M., see Beckett, T.L. (1) 127-138

Nitz, J.A., see Head, E. (4) 1029-1042

Norris, D.G., see Gons, R.A.R. (3) 623-631

Norris, E.H., see Cortes-Canteli, M. (3) 599-608

Nunes, F.C., see Moreira, E.L.G. (2) 495-511

Nunes, J.C., see Moreira, E.L.G. (2) 495-511

O’Brien, J.T., see Mukaetova-Ladinska, E.B. 436

O’Connor, H.M., see Roberts, R.O. (2) 329-339

Oefinger, P.E., see Lachno, D.R. (4) 905-918

Ohta, Y., see Wu, J. (2) 317-328

Okamura, T., see Toda, N. (3) 569-578

Olabarrieta, M., see Choi, S.H. (4) 1043-1049

Olson, S.R., see Wharton, W. (1) 147-156

Olsson, B., C. Malmeström, H. Basun, P. Annas, K. Höglund, L. Lannfelt, N. Andreasen, H. Zetterberg and K. Blennow, Extreme Stability of Chitotriosidase in Cerebrospinal Fluid makes it a Suitable Marker for Microglial Activation in Clinical Trials (2) 273-276

Orimo, S., see Kasahata, N. (4) 889-894

Ostrowski, J., see Gaj, P. (1) 157-168

Pagani, D., see Head, E. (4) 1029-1042

Pallás, M., see del Valle, J. (1) 233-240

Palmer, R.F., see Royall, D.R. (1) 23-32

Palmer, R.F., see Royall, D.R. (2) 467-478

Pamplona, F.A., see Moreira, E.L.G. (2) 495-511

Pankratz, V.S., see Roberts, R.O. (2) 329-339

Parent, B., see Kervern, M. (1) 183-196
Pariente, J., see Wallon, D. (1) 19-22

Pase, M.P., Modifiable Vascular Markers for Cognitive Decline and Dementia: The Importance of Arterial Aging and Hemodynamic Factors (3) 653-663

Pasquier, F., see Richard, F. (3) 765-772

Pasquier, F., see Wallon, D. (1) 19-22

Paziewska, A., see Gaj, P. (1) 157-168

Perluigi, M., see Domenico, F.D. (2) 277-289

Perucho, J., see Solano, R.M. (1) 57-76

Petersen, R.C., see Roberts, R.O. (2) 329-339

Petsko, G., see Bharadwaj, P.R. (4) 949-967

Pfeffer-Baczuk, A., see Gaj, P. (1) 157-168

Pfeifer, A., see Froestl, W. (4) 793-887

Philips, M., see Tales, A. (2) 457-466

Piacentini, S., see Tedde, A. (1) 13-17

Piaceri, I., see Tedde, A. (1) 13-17

Pientka, L., see Polidori, M.C. (3) 521-530

Piguet, O., see Leyton, C.E. (4) 1051-1059

Pleiss, M., see Head, E. (4) 1029-1042

Polidori, M.C., L. Pientka and P. Mecocci, A Review of the Major Vascular Risk Factors Related to Alzheimer's Disease (3) 521-530

Porter, G., see Tales, A. (2) 457-466

Prediger, R.D., see Moreira, E.L.G. (2) 495-511

Price, T.O., see Farr, S.A. (2) 447-455

Puerta, E., see Maioli, S. (2) 341-355

Qi, Y., see Yu, P. (2) 373-385

Qiu, C., Preventing Alzheimer's Disease by Targeting Vascular Risk Factors: Hope and Gap (3) 721-731

Rabkin, S.W., Arterial Stiffness: Detection and Consequences in Cognitive Impairment and Dementia of the Elderly (3) 541-549

Racchi, M., see Lanni, C. (4) 919-926

Ranzani, G.N., see Lanni, C. (4) 919-926

Ranzenigo, A., see Lanni, C. (4) 919-926

Raychaudhuri, M., K. Roy, S. Das and D. Mukhopadhyay, The N-Terminal SH3 Domain of Grb2 is Required for Endosomal Localization of A $\beta$ PP (2) 479-493

Ribeiro-do-Valle, R.M., see Moreira, E.L.G. (2) 495511

Richard, E., E.P. Moll van Charante and W.A. van Gool, Vascular Risk Factors as Treatment Target to Prevent Cognitive Decline (3) 733-740

Richard, F. and F. Pasquier, Can the Treatment of Vascular Risk Factors Slow Cognitive Decline in Alzheimer's Disease Patients? (3) 765-772

Riley, J., see Sanchez, A. (3) 587-597

Rimondini, R., see Maioli, S. (2) 341-355

Ringe, D., see Bharadwaj, P.R. (4) 949-967 
Roberts, L.A., see Roberts, R.O. (2) 329-339

Roberts, R.O., L.A. Roberts, Y.E. Geda, R.H. Cha, V.S. Pankratz, H.M. O'Connor, D.S. Knopman and R.C. Petersen, Relative Intake of Macronutrients Impacts Risk of Mild Cognitive Impairment or Dementia (2) 329-339

Rojas, J.C., A.K. Bruchey and F. Gonzalez-Lima, LowLevel Light Therapy Improves Cortical Metabolic Capacity and Memory Retention (3) 741-752

Rovelet-Lecrux, A., see Wallon, D. (1) 19-22

Roy, K., see Raychaudhuri, M. (2) 479-493

Royall, D.R. and R.F. Palmer, Estimating the Temporal Evolution of Alzheimer's Disease Pathology with Autopsy Data (1) 23-32

Royall, D.R., R.F. Palmer, E.D. Vidoni, R.A. Honea and J.M. Burns, The Default Mode Network and Related Right Hemisphere Structures may be the Key Substrates of Dementia (2) 467-478

Rueckert, D., see Mattila, J. (4) 969-979

Sachs, J., see Wharton, W. (1) 147-156

Saeed, F., see Hashimoto, G. (2) 437-445

Sager, M.A., see Wharton, W. (1) 147-156

Saiki, R., see Waragai, M. (1) 33-41

Sakurai, M., see Hashimoto, G. (2) 437-445

Sanchez, A., D. Tripathy, X. Yin, K. Desobry, J. Martinez, J. Riley, D. Gay, J. Luo and P. Grammas, p38 MAPK: A Mediator of Hypoxia-Induced Cerebrovascular Inflammation (3) 587-597

Sankovich, S.E., see Bharadwaj, P.R. (4) 949-967

Santos, D.B., see Moreira, E.L.G. (2) 495-511

Sasaki, M., see Yasuno, F. (4) 895-903

Savage, S., see Leyton, C.E. (4) 1051-1059

Schall, H.E., see Fawver, J.N. (1) 197-215

Schneider, J.A., see Shah, R.C. (3) 579-586

Schraen, S., see Wallon, D. (1) 19-22

Schwarz, A.J., see Yu, P. (2) 373-385

Segal, S.K., C.W. Cotman and L.F. Cahill, ExerciseInduced Noradrenergic Activation Enhances Memory Consolidation in Both Normal Aging and Patients with Amnestic Mild Cognitive Impairment (4) 1011-1018

Sepulveda-Falla, D., M. Glatzel and F. Lopera, Phenotypic Profile of Early-Onset Familial Alzheimer's Disease Caused by Presenilin-1 E280A Mutation (1) 1-12

Sethuraman, G., see Yu, P. (2) 373-385

Shah, R.C., J.A. Schneider, S. Leurgans and D.A. Bennett, Association of Lower Hemoglobin Level and Neuropathology in Community-Dwelling Older Persons (3) 579-586
Shen, Y., see Wu, J. (2) 317-328

Shi, Y., see Bai, F. (2) 387-396

Siemers, E.R., see Yu, P. (2) 373-385

Silvestrini, M., G. Viticchi, C. Altamura, S. Luzzi, C. Balucani and F. Vernieri, Cerebrovascular Assessment for the Risk Prediction of Alzheimer's Disease (3) 689-698

Sinforiani, E., see Lanni, C. (4) 919-926

Smiley, J.R., see Head, E. (4) 1029-1042

Snowden, R.J., see Tales, A. (2) 457-466

Soininen, H., see Mattila, J. (4) 969-979

Soininen, H., see Tolppanen, A.-M. (3) 531-540

Sol, O., see Fehlbaum-Beurdeley, P. (1) 169-181

Solano, R.M., M.J. Casarejos, A. Gómez, J. Perucho, J.G. de Yébenes and M.A. Mena, Parkin Null Cortical Neuronal/Glial Cultures are Resistant to Amyloid$\beta_{1-42}$ Toxicity: A Role for Autophagy? (1) 57-76

Solomon, A., see Tolppanen, A.-M. (3) 531-540

Son, Y.-D., see Kim, M.-J. (4) 927-938

Sorbi, S., see Tedde, A. (1) 13-17

Stafford, R.B., see Zhang, Q. (3) 677-687

Steele, J.W., see Bharadwaj, P.R. (4) 949-967

Stein, J.H., see Wharton, W. (1) 147-156

Strickland, S., see Cortes-Canteli, M. (3) 599-608

Styczynska, M., see Gaj, P. (1) 157-168

Szybinska, A., see Bialopiotrowicz, E. (2) 397-415

Tabor, A.B., see Barua, N.U. (1) 43-56

Takagi, K., see Waragai, M. (1) 33-41

Talbot, J.A., see Lachno, D.R. (4) 905-918

Tales, A., U. Leonards, A. Bompas, R.J. Snowden, M. Philips, G. Porter, J. Haworth, G. Wilcock and A. Bayer, Intra-Individual Reaction Time Variability in Amnestic Mild Cognitive Impairment: A Precursor to Dementia? (2) 457-466

Tanimukai, S., see Yasuno, F. (4) 895-903

Tashiro, J., see Waragai, M. (1) 33-41

Tauscher, J.T., see Yu, P. (2) 373-385

Tedde, A., I. Piaceri, S. Bagnoli, E. Lucenteforte, S. Piacentini, S. Sorbi and B. Nacmias, DAPK1 is Associated with FTD and not with Alzheimer's Disease (1) 13-17

Teich, A.F., see Hashimoto, G. (2) 437-445

Thomas, F., see Tranah, G.J. (2) 357-372

Toda, N. and T. Okamura, Cerebral Blood Flow Regulation by Nitric Oxide in Alzheimer's Disease (3) $569-578$

Tolppanen, A.-M., A. Solomon, H. Soininen and M. Kivipelto, Midlife Vascular Risk Factors and Alzheimer's Disease: Evidence from Epidemiological Studies (3) 531-540 
Touchon, J., see Fehlbaum-Beurdeley, P. (1) 169-181 Tran, H., see Wolf, A.B. (1) 217-232

Tranah, G.J., M.A. Nalls, S.M. Katzman, J.S. Yokoyama, E.T. Lam, Y. Zhao, S. Mooney, F. Thomas, A.B. Newman, Y. Liu, S.R. Cummings, T.B. Harris, K. Yaffe and for the Health, Aging and Body Composition Study, Mitochondrial DNA Sequence Variation Associated with Dementia and Cognitive Function in the Elderly (2) 357-372

Tripathy, D., see Sanchez, A. (3) 587-597

Uberti, D., see Bialopiotrowicz, E. (2) 397-415

Uchihara, T., see Kasahata, N. (4) 889-894

Uemura, K., see Waragai, M. (1) 33-41

Underbakke, G., see Wharton, W. (1) 147-156

Valla, J., see Wolf, A.B. (1) 217-232

van Dijk, E., see Gons, R.A.R. (3) 623-631

van Dijk, E.J., see van Norden, A.G.W. (3) 667-676

van Gool, W.A., see Richard, E. (3) 733-740

van Norden, A.G.W., I.W.M. van Uden, K.F. de Laat, E.J. van Dijk and F.-E. de Leeuw, Cognitive Function in Small Vessel Disease: The Additional Value of Diffusion Tensor Imaging to Conventional Magnetic Resonance Imaging: The RUN DMC Study (3) 667-676

van Norden, A.G.W., see Gons, R.A.R. (3) 623-631

van Oudheusden, L.J.B., see Gons, R.A.R. (3) 623-631

van Uden, I.W.M., see Gons, R.A.R. (3) 623-631

van Uden, I.W.M., see van Norden, A.G.W. (3) 667676

Vanderstichele, H., see Lachno, D.R. (4) 905-918

Vanrooyen, J., see Head, E. (4) 1029-1042

Velázquez-Zamora, D.A., see del Valle, J. (1) 233-240

Vellas, B., see Fehlbaum-Beurdeley, P. (1) 169-181

Vercelletto, M., see Fehlbaum-Beurdeley, P. (1) 169181

Verdile, G., see Bharadwaj, P.R. (4) 949-967

Vernieri, F., see Silvestrini, M. (3) 689-698

Vidoni, E.D., see Royall, D.R. (2) 467-478

Vieira, D.S.C., see Moreira, E.L.G. (2) 495-511

Villette, V., see Kervern, M. (1) 183-196

Viticchi, G., see Silvestrini, M. (3) 689-698

von Bernhardi, R., see Flores, B. (2) 417-429

Waldemar, G., see Mattila, J. (4) 969-979

Walker, D.G., see Wolf, A.B. (1) 217-232

Wallon, D., A. Rovelet-Lecrux, V. Deramecourt, J. Pariente, S. Auriacombe, I. Le Ber, S. Schraen, F. Pasquier, D. Campion and D. Hannequin, Definite Behavioral Variant of Frontotemporal
Dementia with C9ORF72 Expansions Despite Positive Alzheimer's Disease Cerebrospinal Fluid Biomarkers (1) 19-22

Walz, R., see Moreira, E.L.G. (2) 495-511

Wang, X., Q. Chen and D. Xing, Focal Adhesion Kinase Activates NF- $\kappa \mathrm{B}$ via the ERK1/2 and p38MAPK Pathways in Amyloid- $\beta_{25-35}$-Induced Apoptosis in PC12 Cells (1) 77-94

Wang, Z., see Zhang, Q. (3) 677-687

Waragai, M., M. Yoshida, M. Mizoi, R. Saiki, K. Kashiwagi, K. Takagi, H. Arai, J. Tashiro, M. Hashimoto, N. Iwai, K. Uemura and K. Igarashi, Increased Protein-Conjugated Acrolein and Amyloid- $\beta_{40 / 42}$ Ratio in Plasma of Patients with Mild Cognitive Impairment and Alzheimer's Disease (1) 33-41

Webb, R.L., see Beckett, T.L. (1) 127-138

Welser, K., see Barua, N.U. (1) 43-56

Weng, Y.-Q., see Girão da Cruz, M.T. (2) 267-272

Wharton, W., J.H. Stein, C. Korcarz, J. Sachs, S.R. Olson, H. Zetterberg, M. Dowling, S. Ye, C.E. Gleason, G. Underbakke, L.E. Jacobson, S.C. Johnson, M.A. Sager, S. Asthana and C.M. Carlsson, The Effects of Ramipril in Individuals at Risk for Alzheimer's Disease: Results of a Pilot Clinical Trial (1) 147-156

Wilcock, G., see Tales, A. (2) 457-466

Willis, B.A., see Yu, P. (2) 373-385

Wise-Scira, O., see Fawver, J.N. (1) 197-215

Wojda, U., see Bialopiotrowicz, E. (2) 397-415

Wolf, A.B., B.B. Braden, H. Bimonte-Nelson, Y. Kusne, N. Young, E. Engler-Chiurazzi, A.N. Garcia, D.G. Walker, G.S.D. Moses, H. Tran, F. LaFerla, L. Lue, N.E. Lombardo and J. Valla, Broad-Based Nutritional Supplementation in 3xTg Mice Corrects Mitochondrial Function and Indicates Sex-Specificity in Response to Alzheimer's Disease Intervention (1) 217-232

Wolk, D.A., see Zhang, Q. (3) 677-687

Wolz, R., see Mattila, J. (4) 969-979

Wu, J., J. Yang, Y. Yu, Q. Li, N. Nakamura, Y. Shen, Y. Ohta, S. Yu and K. Abe, Delayed Audiovisual Integration of Patients with Mild Cognitive Impairment and Alzheimer's Disease Compared with Normal Aged Controls (2) 317-328

Wurster, I., see Maetzler, W. (1) 95-100

Wyatt, M.J., see Barua, N.U. (1) 43-56

Xekardaki, A., see Costanza, A. (3) 643-652

Xing, D., see Wang, X. (1) 77-94

Xiong, Y., see Zhao, Q. (4) 1019-1027 
Xu, K., see Huang, H.-C. (4) 981-996

Xu, X., see Bai, F. (2) 387-396

Yaffe, K., see Tranah, G.J. (2) 357-372

Yamashita, F., see Yasuno, F. (4) 895-903

Yang, J., see Wu, J. (2) 317-328

Yasuno, F., S. Tanimukai, M. Sasaki, C. Ikejima, F. Yamashita, C. Kodama, K. Mizukami and T. Asada, Combination of Antioxidant Supplements Improved Cognitive Function in the Elderly (4) 895-903

Ye, S., see Wharton, W. (1) 147-156

Yin, X., see Sanchez, A. (3) 587-597

Yokoyama, J.S., see Tranah, G.J. (2) 357-372

Yoshida, M., see Waragai, M. (1) 33-41

Young, N., see Wolf, A.B. (1) 217-232

Ypsilanti, A., see Girão da Cruz, M.T. (2) 267-272

Yu, P., R.A. Dean, S.D. Hall, Y. Qi, G. Sethuraman, B.A. Willis, E.R. Siemers, F. Martenyi, J.T. Tauscher, A.J. Schwarz and for the Alzheimer's Disease Neuroimaging Initiative, Enriching Amnestic Mild Cognitive Impairment Populations for Clinical Trials: Optimal Combination of Biomarkers to Predict Conversion to Dementia (2) 373-385

Yu, S., see Wu, J. (2) 317-328

Yu, Y., see Wu, J. (2) 317-328
Yuan, Y., see Bai, F. (2) 387-396

Yue, C., see Bai, F. (2) 387-396

Yue, Z., see Bharadwaj, P.R. (4) 949-967

Zajicek, J.P., see Escudero, J. (4) 997-1010

Zamolodchikov, D., see Cortes-Canteli, M. (3) 599608

Zetterberg, H., see Olsson, B. (2) 273-276

Zetterberg, H., see Wharton, W. (1) 147-156

Zhang, Q., R.B. Stafford, Z. Wang, S.E. Arnold, D.A. Wolk and J.A. Detre, Microvascular Perfusion Based on Arterial Spin Labeled Perfusion MRI as a Measure of Vascular Risk in Alzheimer's Disease (3) 677-687

Zhang, Z., see Bai, F. (2) 387-396

Zhao, Q., Y. Xiong, D. Ding, Q. Guo and Z. Hong, Synergistic Effect between Apolipoprotein E $\varepsilon 4$ and Diabetes Mellitus for Dementia: Result from a Population-Based Study in Urban China (4) 10191027

Zhao, Y., see Tranah, G.J. (2) 357-372

Zhou, W., see Fehlbaum-Beurdeley, P. (1) 169-181

Zhu, X., see Fawver, J.N. (1) 197-215

Zhuang, L., see Bai, F. (2) 387-396

Zwiers, M.P., see Gons, R.A.R. (3) 623-631 